\title{
Assisted Reproductive Technologies and Infectious Diseases
}



Andrea Borini - Valeria Savasi

Editors

\section{Assisted Reproductive Technologies and Infectious Diseases}

A Guide to Management

照 Springer 
Editors

Andrea Borini, MD

Scientific Director

9.baby

Center for Fertility and Family

Health

Bologna, Italy
Valeria Savasi, MD, PhD

Chief of Reproductive Unit

Unit of Obstetrics and

Gynecology

Department of Biomedical and Clinical Sciences

Hospital "L. Sacco"

University of Milan

Milan, Italy

ISBN 978-3-319-30110-5

ISBN 978-3-319-30112-9 (eBook)

DOI 10.1007/978-3-319-30112-9

Library of Congress Control Number: 2016943072

(C) Springer International Publishing Switzerland 2016

This work is subject to copyright. All rights are reserved by the Publisher, whether the whole or part of the material is concerned, specifically the rights of translation, reprinting, reuse of illustrations, recitation, broadcasting, reproduction on microfilms or in any other physical way, and transmission or information storage and retrieval, electronic adaptation, computer software, or by similar or dissimilar methodology now known or hereafter developed.

The use of general descriptive names, registered names, trademarks, service marks, etc. in this publication does not imply, even in the absence of a specific statement, that such names are exempt from the relevant protective laws and regulations and therefore free for general use.

The publisher, the authors and the editors are safe to assume that the advice and information in this book are believed to be true and accurate at the date of publication. Neither the publisher nor the authors or the editors give a warranty, express or implied, with respect to the material contained herein or for any errors or omissions that may have been made.

Printed on acid-free paper

This Springer imprint is published by Springer Nature

The registered company is Springer International Publishing AG Switzerland 


\section{Preface}

The idea of a book focusing on the infectious diseases and assisted reproductive technologies (ART) was born 3 years ago because reproductive assistance in subjects infected with blood viruses is still a complex issue. When we sat down to plan the first edition of this book, we were determined to make it innovative, comprehensive and accessible. We contacted the most important researchers in infectious diseases and ART in the world, and we asked them if they would be interested in participating in this project.

The infectious diseases involved in ART are various, but we decided to concentrate our attention on only some of them, and we chose the viral infections. Viral infections are the most interesting infections because the viruses are changeable, unpredictable, and most are untreatable. The word virus has a much longer history than the study of what we now call by that name. It comes directly from the Latin virus, a term meaning, "poison, sap of plants, slimy liquid". Its earliest known use in English to denote a disease-causing agent was in 1728 , although for the rest of the eighteenth century, throughout the nineteenth and for several decades beyond, there was no clear distinction between virus as a vague term, applicable to any infectious microbe, and the very particular group of entities we know as viruses today. Moreover, some viruses create chronic infectious diseases: the most important are human immunodeficiency virus-1 (HIV-1), hepatitis $\mathrm{C}$ virus (HCV) and hepatitis $\mathrm{B}$ virus (HBV) infections. All of these chronic infections are ubiquitous and very dangerous because each of these viruses can lead to a widespread outbreak. Some outbreaks of viral disease quickly escalate, depending on viral transmissibility and virulence. These are the 
crucial parameters. For the blood-borne viruses, transmission is more complicated than for other types of virus. Generally, it depends on a third party: a vector. The viruses have to replicate in the blood of the host to produce severe viraemia. The vector-an insect, for example-must arrive for a meal, bite the host, slurp up the virions along with the blood, and carry them away. The yellow fever virus transmits this way. However, blood-borne viruses can also spread to new hosts by way of needles. Ebola, HIV and HCV, three viruses of very different characters and very different adaptive strategies, all happen to move well via needles. Sexual transmission is a good scheme for viruses with a low degree of hardiness in the external environment. Transmission during coitus is a conservative strategy, avoiding the risk of air or sun contact. Whatever the case, the sexually transmitted viruses tend towards patience. They cause persistent infections and endure long periods of latency (e.g. the herpes virus), or they replicate slowly (as with HIV and HBV).

In the last few years, clinicians have learned to treat an HIV infection with drugs, an HBV infection with a vaccine, an HVC infection with some drugs, and this situation has opened the door for the patient's desire to have children. Even in the presence of a chronic infection, or probably because the infection is chronic and well controlled, people need to have progeny. For this reason, clinicians have to address the patient's requests regarding pregnancies, and they have to try to save pregnancies and newborn babies. HBV infection seems to have minor relevance because a vaccine is available, but in the very near future the HBV virus will be able to change; if that happens, clinicians will be able to assist their infected patients. For the HIV virus, we have to consider that three quarters of individuals infected with it are in their reproductive years and may consider pregnancy planning. Techniques have been developed that can minimise the risk of HIV transmission in these couples, and ART programmes should be integrated into global public health services against HIV.

Regarding the HCV virus, the debate on HCV-discordant couples requiring ART is still open. Sexual transmission of HCV is a controversial issue, whereas the presence of the virus in the sperm has already been demonstrated. In ART, HCV transmission raises 
some questions. One of these is that specific guidelines regarding the behaviour of physicians in reproductive medicine have not yet been established. Thus, this book will try to answer many of the questions about these issues, obtaining a picture of the biological aspects of HIV, HCV and HBV infections and the real data presented in the literature regarding reproductive aspects in their presence. Finally, there is a chapter discussing the reproductive possibilities in poorer countries in Africa and South America, where these infectious diseases are more prevalent.

It is our hope that this resource will be of assistance to physicians and scientists engaged in this exciting field of medicine.

Bologna, Italy

Andrea Borini

Milan, Italy

Valeria Savasi 



\section{Contents}

1 The Impact of Human Immunodeficiency Virus (HIV) and Hepatitis B Virus (HBV) and Hepatitis C Virus (HCV) on Male and Female Fertility

Elisabeth van Leeuwen

2 HBV Virus in the Future

Gianguglielmo Zehender, Erika Ebranati, Lisa Fiaschi, Massimo Ciccozzi, and Massimo Galli

3 Reproductive Assistance in HIV-Serodiscordant Couples Where the Man Is Positive.

Rocío Rivera, $\mathrm{M}^{\mathrm{a}}$ Carmen Galbis, and Nicolás Garrido Puchalt

4 Reproductive Assistance in HIV-Serodiscordant Couples Where the Woman Is Positive

Daniel Mataró, Rita Vassena, Oriol Coll, and Valérie Vernaeve

5 The Presence of Hepatitis B and C Virus in Human Gametes and Embryos

Xiao-Ling Hu, Jia-Li You, Hui-Hui Pan, Miao Li, and Yi-Min Zhu

6 Detection of Hepatitis C Virus in the Semen of Infected Men and Reproductive Assistance in HCV Discordant Couples: An Overview

Valeria Savasi and Luca Mandia 
x Contents

7 Laboratory Safety During Assisted Reproduction in Patients with a Bloodborne Virus

Asma Sassi, F. Devreker, and Yvon Englert

8 Accessibility to Reproductive Assistance in Low-Income Countries.

Irene Cetin and Arianna Laoreti

Index

203 


\section{Contributors}

Irene Cetin, MD Unit of Obstetrics and Gynecology, Department of Mother and Child, Luigi Sacco, Milan, Italy

Massimo Ciccozzi, MD, PhD Dipartimento Malattie Infettive, Parassitarie ed Immunomediate, Istituto Superiore di Sanità, Rome, Italy

Oriol Coll, MD, PhD Clinica Eugin, Barcelona, Spain

Fabienne Devreker, MD, PhD Laboratory for Research in Human Reproduction, Medicine Faculty, Department of Obstetrics and Gynecology, Hôpital Erasme, Université Libre de Bruxelles (ULB), Brussels, Belgium

Erika Ebranati, PhD Dipartimento di Scienze Cliniche e Biomediche "Luigi Sacco", Sezione di Malattie Infettive, Università degli Studi di Milano, Milan, Italy

Rocio Rivera, MSc Andrology Laboratory, Instituto Universitario IVI Valencia, Valencia, Spain

Yvon Englert, MD, PhD, MBA Laboratory for Research in Human Reproduction, Medicine Faculty, Department of Obstetrics and Gynecology, Hôpital Erasme, Université Libre de Bruxelles (ULB), Brussels, Belgium

$\mathbf{M}^{\mathrm{a}}$ Carmen Galbis, PhD, MSc Andrology Laboratory, Instituto Universitario IVI Valencia, Valencia, Spain

Ophthalmology Investigation, Hospital Universitario Dr. Peset, Valencia, Spain 
Lisa Fiaschi Dipartimento di Scienze Cliniche e Biomediche "Luigi Sacco", Sezione di Malattie Infettive, Università degli Studi di Milano, Milan, Italy

Massimo Galli Dipartimento di Scienze Cliniche e Biomediche "Luigi Sacco", Sezione di Malattie Infettive, Università degli Studi di Milano, Milan, Italy

Xiao-Ling Hu, PhD Department of Reproductive Endocrinology, Women's Hospital, School of Medicine, Zhejiang University, Hangzhou, China

Arianna Laoreti, MD Unit of Obstetrics and Gynecology, Department of Mother and Child, Luigi Sacco, Milan, Italy

Elisabeth van Leeuwen, MD, PhD Department of Obstetrics and Gynecology, Academic Medical Center, Amsterdam, The Netherlands

Miao Li, MD Department of Reproductive Endocrinology, Women's Hospital, School of Medicine, Zhejiang University, Hangzhou, China

Luca Mandia, MD Department of Gynecology and Obstetrics, University of Milan, Milan, Italy

Daniel Mataró, MD, PhD Clinica Eugin, Barcelona, Spain

Hui-Hui Pan Department of Reproductive Endocrinology, Women's Hospital, School of Medicine, Zhejiang University, Hangzhou, China

Nicolás Garrido Puchalt, PhD, MSc Andrology Laboratory, Instituto Universitario IVI Valencia, Valencia, Spain

Asma Sassi, MD Laboratory for Research in Human Reproduction, Medicine Faculty, Department of Obstetrics and Gynecology, Hôpital Erasme, Université Libre de Bruxelles (ULB), Brussels, Belgium 
Valeria Savasi, MD, PhD Unit of Obstetrics and Gynecology, Department of Biomedical and Clinical Sciences, Hospital "L. Sacco", University of Milan, Milan, Italy

Rita Vassena, DVM, PhD Clinica Eugin, Barcelona, Spain

Valérie Vernaeve, MD, PhD Clinica Eugin, Barcelona, Spain

Jia-Li You, MD Department of Reproductive Endocrinology, Women's Hospital, School of Medicine, Zhejiang University, Hangzhou, China

Valley Medical Oncology Consultants, Mountain View, CA, USA

Gianguglielmo Zehender, PhD Dipartimento di Scienze Cliniche e Biomediche "Luigi Sacco", Sezione di Malattie Infettive, Università degli Studi di Milano, Milan, Italy

Yi-Min Zhu, PhD Department of Reproductive Endocrinology, Women's Hospital, School of Medicine, Zhejiang University, Hangzhou, China 